\title{
Thyroid and Heart
}

\author{
Robin Maskey, Additional Professor, \\ Department of Internal Medicine, BPKIHS, Dharan, Nepal
}

\section{INTRODUCTION}

The common signs and symptoms of thyroid disease are due to the effects of thyroid hormone on the heart and cardiovascular system. ${ }^{1}$ Both hyperthyroidism and hypothyroidism produce changes in cardiac contractility, myocardial oxygen consumption, cardiac output, blood pressure, and systemic vascular resistance $(\mathrm{SVR})^{2}$, which are reversible when the underlying thyroid disorder is treated.

The thyroid gland primarily secretes $\mathrm{T} 4(85 \%)$, which is converted to T3 by 5 -monodeiodination in the liver, kidney, and skeletal muscle. ${ }^{3}$ The heart relies mainly on serum $\mathrm{T} 3$ because no significant myocyte intracellular deiodinase activity takes place, and it appears that $\mathrm{T} 3$, and not $\mathrm{T} 4$, is transported into the myocyte.

\section{Effects of Thyroid Hormone on Cardiovascular Hemodynamics}

Thyroid hormone mediates the expression of both structural and regulatory genes in the cardiac myocyte, ${ }^{4}$ includes sarcoplasmic reticulum Ca2ATPase and its inhibitor phospholamban (which regulate the uptake of calcium into the sarcoplasmic reticulum during diastole). ${ }^{4}$ In the VSM cell, thyroid hormone mediated effects are due to both genomic (T3 is binding to TRs, which regulate transcription of specific cardiac genes) and nongenomic actions (direct modulation of membrane ion channels).

\section{Hyperthyroidism}

In hyperthyroidism, cardiac contractility is enhanced, and resting heart rate and $50 \%$ to $300 \%$ higher cardiac output than normal individuals ${ }^{5}$ because of increasing blood volume and preload stimulated by $\mathrm{T} 3$ via synthesis of renin substrate in the liver. ${ }^{6}$ The exercise intolerance occurs because inability to increase heart rate and ejection fraction or lower SVR and skeletal muscle weakness may be the predominant cause in long standing disease or elderly.

Sinus tachycardia is the most common rhythm disturbance which predisposes to atrial fibrillation because T3 increases systolic depolarization and diastolic repolarization, and decreases the action potential duration, the refractory period of the atrial myocardium, and the atrial/ventricular nodal refractory period. It appears that subclinical (mild) hyperthyroidism carries the same relative risk for atrial fibrillation as does overt disease. Rarely patients with hyperthyroidism develop chest pain and EKG changes suggestive of cardiac ischemia. ${ }^{7}$ Severe hyperthyroidism leads to high-output HF in preexistent ischemic or hypertensive heart disease and even in patients without underlying heart disease.4Overt and SHyper have been associated with increased markers of thrombogenesis (fibrinogen and factor X levels).

Treatment of atrial fibrillation in the setting of hyperthyroidism can be obtained by oral beltablockers and role of anticoagulation of patients is controversial. ${ }^{1}$

\section{Hypothyroidism}

In hypothyroidism, endothelial dysfunction and impaired VSM relaxation lead to increased $\mathrm{SVR}^{8}$ leading to diastolic hypertension in $30 \%$ of patients, and thyroid hormone replacement therapy restores endothelial-derived vasorelaxation and blood pressure to normal in most. It also causes a prolongation of the QT interval that predisposes the patient to ventricular irritability and torsade 
which is reversible by treatment. The genomic changes explain the physiological changes such as the slowing of the isovolumic relaxation phase of diastolic function characteristic of hypothyroidism and are responsive to $\mathrm{T} 4$ replacement.

Hyperlipidemia in hypothyroidism is due to a decrease in LDL receptors, resulting in reduced cholesterol clearance from the liver and decreased activity of cholesterol ${ }^{7}$ a-hydroxylase, which is activated by TH, in breaking down cholesterol. LT4 repalcement is more effective in dyslipidemia in $\mathrm{SCH}$ when total cholesterol $>240$ than $<240 \mathrm{mg} / \mathrm{dl}$.

\section{References}

1. Klein I, Ojamaa K. Thyroid hormone and the cardiovascular system. N Engl J Med. 2001;344:501509.

2. Kahaly GJ, Dillmann WH. Thyroid hormone action in the heart. Endocrine Rev. 2005;26:704-728.
3. Maia AL, Kim BW, Huang SA, Harney JW, Larsen PR. Type 2 iodothyronine deiodinase is the major source of plasma T3 in euthyroid humans. J Clin Invest. 2005;115:2524-2533.

4. Dillmann WH. Cellular action of thyroid hormone on the heart. Thyroid. 2002;12:447-452.

5. Danzi S, Klein I. Thyroid hormone and the cardiovascular system. Minerva Endocrinologica. 2004;29:139-150.

6. Laragh JH, Sealey JE. Relevance of the plasma renin hormonal control system that regulates blood pressure and sodium balance for correctly treating hypertension and for evaluating ALLHAT. Am J Hypertens. 2003;16:407-415.

7. Choi YH, Chung JH, Bae SW, Lee WH, Jeong EM, Kang MG, Kim BJ, Kim KW, Park JE. Severe coronary artery spasm can be associated with hyperthyroidism. Coron Artery Dis. 2005;16:135-139.

8. Napoli R, Biondi B, Guardasole V, Matarazzo M, Pardo F, Angelini V, Fazio S, Sacca L. Impact of hyperthyroidism and its correction on vascular reactivity in humans. Circulation. 2001;104:30763080 . 